\title{
The influence of latent toxoplasmosis on women's reproductive function: four cross-sectional studies
}

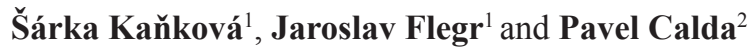 \\ ${ }^{1}$ Department of Philosophy and History of Science, Faculty of Science, Charles University, Prague, Czech Republic; \\ ${ }^{2}$ Department of Obstetrics and Gynaecology, General University Hospital and First Faculty of Medicine, Charles University, Prague, \\ Czech Republic
}

\begin{abstract}
Several studies have investigated the association between infection with Toxoplasma gondii (Nicolle et Manceaux, 1908), pregnancy and fertility, but the results of studies focused on the fertility are rather ambiguous. Here we report results of four new cross-sectional studies. The studies were performed in the General University Hospital, Prague (study A with n $=1165$, and study C with $n=317$ ), in private clinics of the Centre of Reproductive Medicine, Prague (study B with $n=1016$ ), and in a population of Czech and Slovak volunteers from the Facebook page 'Guinea Pigs' willing to participate in various basic science studies (study D with $\mathrm{n}=524$ ). In studies $\mathrm{A}$ and $\mathrm{B}$, the clinical records were used to assess the fertility problems, whereas in studies $\mathrm{C}$ and $\mathrm{D}$, the women were asked to rate their fertility problems using a six-point scale. Pregnant $T$. gondii-infected women were older than $T$. gondii-free women (study A: 33.1 vs 31.2, $\mathrm{P}<0.001$; study B: 30.6 vs 29.6, $\mathrm{P}=0.012$ ) and more often used assisted reproductive technology to conceive (study A: $17.2 \%$ vs $12.4 \%, \mathrm{P}=0.041$; study B: $13.4 \%$ vs $9.2 \%, \mathrm{P}=0.317$ ). Pregnant $T$. gondii-infected primiparous women were older than T. gondii-free primiparas (study A: $31.1 v s 29.5, \mathrm{P}<0.001$; study B: $29.7 v s 28.9, \mathrm{P}=0.064$ ) and more often used assisted reproductive technology to conceive (study A: $24.7 \%$ vs $14.4 \%, \mathrm{P}=0.010$; study B: $15.9 \%$ vs $15.5 \%, \mathrm{P}=0.888$ ). T. gondii-infected women reported to take a longer time to conceive than $T$. gondii-free women $(\mathrm{P}=0.015)$. They also claimed to have more fertility problems than $T$. gondii-free women $(\mathrm{P}<0.0001)$. Our results suggest that 'asymptomatic' latent toxoplasmosis could be a more serious source of fertility problems and health-associated burden than more severe but far rarer congenital toxoplasmosis.
\end{abstract}

Keywords: parasite, Toxoplasma gondii, infertility, age, manipulation hypothesis

Toxoplasmosis, probably the most widespread human parasitic infectious disease in developed countries, is caused by a coccidian protist, Toxoplasma gondii (Nicolle et Manceaux, 1908). The prevalence of latent toxoplasmosis in the population ranges from $20 \%$ to $80 \%$ depending on various environmental and socioeconomic factors, including the number of cats in the environment, latitude, moisture, hygienic standards and kitchen habits. Toxoplasmosis is transmitted to humans by ingesting food or water contaminated with cat faeces or by eating undercooked meat containing tissue cysts of the parasite (Tenter et al. 2000). Acute disease promoted by rapidly dividing tachyzoites in cells of many tissues usually progresses spontaneously to the latent phase characterised by probably permanent presence of slowly dividing bradyzoites in tissue cysts in various organs of the host. Latent toxoplasmosis is mostly considered to pose no health threat to immunocompetent individuals. However, it is accompanied by specific changes in the psychomotor performance, behaviour and personality profile (Flegr et al. 1996, Lindová et al. 2006, Flegr 2013a).
The clinical implications of $T$. gondii infection in pregnant women are manifold. Unlike acute toxoplasmosis in pregnancy, which could result in congenital toxoplasmosis and serious damages to foetuses and newborns (Wolf et al. 1939, Tenter et al. 2000), latent toxoplasmosis of the mother seems to have no significant negative impact on the health of the offspring. However, pregnant women with latent toxoplasmosis have been reported to have prolonged pregnancy and seemingly younger (less developed) foetuses, especially at the $16^{\text {th }}$ week of pregnancy (Flegr et al. 2005, Kaňková and Flegr 2007). A hypothesis of relaxed quality control explains these phenomena by postulating that $T$. gondii could relax the stringency of some 'quality control' mechanism, which, under normal conditions, is responsible for miscarriage of embryos with developmental defects and thus with a (statistically) slower foetal growth (Kaňková and Flegr 2007). This hypothesis can also explain an earlier observation of extremely high prevalence of toxoplasmosis in mothers of children with Down syndrome (84\% vs 32\% in controls - Hostomská et al. 1957). The hypothesis of relaxed quality control was

Address for correspondence: Š. Kaňková, Department of Philosophy and History of Science, Charles University, Viničná 7, 128 44 Prague 2, Czech Republic. Phone: +420 221951821; Fax:+420 224919704; E-mail: kankova.sarka@gmail.com 
later supported both in humans (Kaňková et al. 2007a) and experimentally infected mice (Kaňková et al. 2007b). The secondary sex ratio was higher in children of women with latent toxoplasmosis than in those of $T$. gondii-free women. Similarly, mice with toxoplasmosis had a higher sex ratio (expressed as the proportion of males in the offspring) than controls in the early phase of latent infection. These phenomena can be explained by a decreased miscarriage rate due to more immunogenic male embryos (KellokumpuLehtinen and Pelliniemi 1984, Christiansen et al. 2004).

The influence of toxoplasmosis on the miscarriage rate in humans has been the subject of several studies yielding discordant results. A positive effect of latent toxoplasmosis on the risk of miscarriage has been shown in a study performed on 5033 obstetric patients (Kimball et al. 1971); however, this observation was not confirmed by others (Giorgino and Mega 1981, Quablan et al. 2002). Pavlinová et al. (2011) have reported the seroprevalence of IgG antibodies against $T$. gondii in 530 women with recurrent miscarriage to be $42.1 \%$, which was significantly higher than in controls (25.1\%). Salman (2014) has shown a significant relationship between toxoplasmosis and anti-Mullerian hormone level as a predictor of ovarian reserve in females.

Recently, latent toxoplasmosis has been shown to be associated with an increase in autoimmune thyroid diseases in pregnancy (Kaňková et. al 2014) and gestational diabetes mellitus (our unpubl. data). The main purpose of the present study is to search for indices of negative effects of latent toxoplasmosis on female fertility. We found that $T$. gondii-infected women became pregnant at an older age, more often needed in vitro fertilisation and reported to take a longer time to conceive and to have more fertility problems than $T$. gondii-negative women.

\section{MATERIALS AND METHODS}

\section{Patients}

Study A was designed as a cross-sectional study. The main data set covered 1165 women who were routinely screened in gestational weeks 9-12 for chromosomal abnormalities (i.e. serum free beta-hCG and PAPP-A) in the General University Hospital in Prague, Czech Republic in 2008-2009. Serum samples collected at the time of screening were frozen and stored at $-70^{\circ} \mathrm{C}$ and 3-4 years later were examined for anamnestic antibodies against Toxoplasma gondii.

The second experimental set (study B) consisted of clients of two outpatient clinics (Centre of Reproductive Medicine) in Prague 5 and Prague 8. We analysed anonymised clinical data on the progress of 1016 pregnancies. The original data set included records of all clients tested for toxoplasmosis from 1996-2004 who were Czech citizens domiciled in Prague. The women were tested for toxoplasmosis at about pregnancy week 16.

Clinical records comprised maternal age, maternal pre-pregnancy weight, number of previous deliveries, and method of conception (in vitro fertilisation $v s$ natural conception) in both data sets and antibody titres in the second data set. The women with incomplete medical records were excluded from the analyses.

The third data set (study C) included information from a questionnaire survey performed in 2012-2013 in women involved in study A. For various technical reasons, the questionnaire only reached about 1200 women. The respondents were asked, among other questions unrelated to the present study, how long it took them to conceive. They were instructed to rate this period using a six-point scale: 1 - less than three months, 2 - from three to six months, 3 - from six to nine months, 4 - from nine to 12 months, 5 - from 12 to 24 months, and 6 - more than 24 months. Other options were "I do not remember" or "this was an unplanned pregnancy". The information about the time to conceive was provided by 317 women.

During the whole time of the studies, we worked with an anonymised data set. The studies were approved by the Ethical Committee of the General University Hospital and the First Faculty of Medicine of the Charles University in Prague.

The fourth set of data (study D) was obtained using an electronic questionnaire distributed as a Qualtrics survey. The subjects were recruited for a study concerning primarily the effects of keeping dogs and cats on personality and health using a Facebook-based snowball method, namely through an invitation posted on the wall of the Facebook page 'Guinea pigs' intended for Czech and Slovak nationals willing to take part in diverse, mostly evolutionary psychology surveys and experiments (www. facebook.com/pokusnikralici). Among 180 questions, we asked the respondents about their gender, age and fertility problems. To rate the fertility problems, they indicated either "I have not check it yet" or used a six-point scale anchored with 1 - not at all and 6 - serious or frequent. The participants were informed about the aims of the study on the first page of an electronic questionnaire. They provided information that the study was voluntary and anonymous, the obtained data would be used exclusively for scientific purposes, and the participants were free to withdraw from the study at any time by closing the web page. This study was approved by the Institutional Review Board (IRB) of the Faculty of Science, Charles University.

\section{Immunological tests for toxoplasmosis}

The complement-fixation test (CFT), which determines the overall levels of IgM and IgG antibodies of particular specificity, and enzyme-linked immunosorbent assay (ELISA) (IgG ELISA: SEVAC, Prague, IgM ELISA: TestLine, Brno) were used to detect toxoplasmosis status of women in studies A and C. By using both IgM and IgG ELISA, we were able to discriminate between the acute and latent phases of the infection. Latent toxoplasmosis was defined as a CFT titre of $1: 4$ and more, together with an index of positivity (IP) of $>1.2$ for IgG ELISA and IP of $<1.0$ for IgM ELISA (Kodym et al. 2007). The presence of anamnestic antibodies against $T$. gondii was diagnosed with the indirect immunofluorescence test at dilutions between $1: 8$ and $1: 1024$ in the second data set (study B). The samples with specific fluorescence visible in a dilution $1: 16$ or higher were considered as $T$. gondii positive. Women with suspected acute toxoplasmosis, i.e. with high IgM and IgA titres, were excluded from the studies (study A: 2 cases; study B: 2 cases).

\section{Statistics}

The statistical program Statistica 9.0 was used for all testing. The influence of latent toxoplasmosis on the age at pregnancy was evaluated using the General Linear Model (GLM), with maternal age at pregnancy as a dependent variable and $T$. gondii 
Table 1. Differences in the time to conceive between Toxoplasma gondii-negative and Toxoplasma gondii-positive women (study C).

\begin{tabular}{lccccccc}
\hline Time to conceive (months) & $<3$ & $3-6$ & $6-9$ & $9-12$ & $12-24$ & Total number* \\
\hline Toxoplasma gondi-negative & $135^{*}$ & 37 & 14 & 16 & 18 & 24 \\
Toxoplasma gondi-positive & 33 & 9 & 242 & 6 & 14 & 32 \\
\hline Total & 168 & 46 & 16 & 22 & 33 \\
\hline
\end{tabular}

* number of women.

status (negative/positive) as an independent binary variable. The number of previous pregnancies was included in all statistical analyses as an independent ordinal variable. The influence of the binary variable latent toxoplasmosis on the probability of having a baby using assisted reproductive technology was evaluated using the contingency table method. For some women, certain data (e.g. number of previous pregnancies and method to conceive) were not available and therefore the numbers of women varied between analyses.

Differences in the time to conceive between $T$. gondii-negative and $T$. gondii-positive women were analysed using the non-parametric Spearman correlation. The mothers who reported either unplanned pregnancy $(n=35)$ or did not remember the time to conceive $(n=7)$ were excluded from the analysis.

The association between toxoplasmosis and fertility problems was analysed by the partial Kendall's tau correlation test with age as a confounding variable (Siegel and Castellan 1988, Kaňková et al. 2011). The Excel sheet for the computation of the partial Kendall correlation is available at http://web.natur.cuni.cz/ flegr/ programy.php.

\section{RESULTS}

The first data set (study A) included 1165 pregnant women of whom 267 tested Toxoplasma gondii positive (prevalence of 22.9\%). Information about the number of the previous pregnancies was not available for 241 women. GLM with the mother's age as a dependent variable and toxoplasmosis and the number of previous pregnancies as independent variables showed that $214 T$. gondii-positive women were older when pregnant $($ mean $=32.1$ years; $\mathrm{CI}_{95}=31.5$, 32.6) than $710 \mathrm{~T}$. gondii-negative women $\left(\right.$ mean $=30.9$ years; $\left.\mathrm{CI}_{95}=30.6,31.2\right)(\mathrm{F}=14.66, \mathrm{P}<0.001$, $\left.\mathrm{eta}^{2}=0.016\right)$. The effect of the second predictor 'number of previous pregnancies' on the mother's age was positive and also significant $\left(\mathrm{F}=142.55, \mathrm{P}<0.001, \mu^{2}=0.134\right)$. Next, the influence of latent toxoplasmosis on the mother's age at pregnancy was evaluated in a subset of primiparous women. This analysis showed that $113 \mathrm{~T}$. gondiipositive primiparas were significantly older when pregnant (mean $=31.1$ years; $\left.\mathrm{CI}_{95}=30.4,31.7\right)$ than $346 \mathrm{~T}$. gondiinegative primiparas (mean $=29.5$ years; $\left.\mathrm{CI}_{95}=29.0,29.9\right)$ $\left(\mathrm{F}=12.26, \mathrm{P}<0.001, \mu^{2}=0.026\right)$.

Differences in the frequency of in vitro fertilisation between $T$. gondii-negative and $T$. gondii-positive women were analysed for 1165 women from study A. Assisted reproductive technology (ART), mostly in vitro fertilisation, was used by 111 (12.4\%) of $898 \mathrm{~T}$. gondii-negative women in comparison with $46(17.2 \%)$ of $267 \mathrm{~T}$. gondiipositive women. The difference was significant $\left(\chi^{2}=4.18\right.$, $\mathrm{P}=0.041, \mathrm{OR}=1.48)$. The same analysis was repeated in primiparas. Again, the frequency of using ART was low- er in T. gondii-negative primiparous women (47 of 327, $14.4 \%$ ) than in $T$. gondii-positive primiparous women (27 of $109,24.7 \%),\left(\chi^{2}=6.27, \mathrm{P}=0.010, \mathrm{OR}=1.96\right)$.

The second data set (study B) consisted of 1016 pregnant women of whom 201 were $T$. gondii-positive (prevalence of $19.8 \%$ ). The GLM showed that $201 \mathrm{~T}$. gondiipositive women were significantly older at pregnancy $\left(\right.$ mean $=30.6$ years; $\left.\mathrm{CI}_{95}=30.0,31.2\right)$ than 815 T. gondiinegative women $\left(\right.$ mean $=29.6$ years; $\left.\mathrm{CI}_{95}=29.3,29.9\right)$ $\left(\mathrm{F}=6.4, \mathrm{P}=0.012, \mu^{2}=0.006\right)$. The effect of the second predictor, i.e. the number of previous pregnancies, was also significant $\left(\mathrm{F}=101.96, \mathrm{P}<0.001, \mu^{2}=0.091\right)$. Further, the analysis showed that $113 \mathrm{~T}$. gondii-positive primiparas were older when pregnant $\left(\right.$ mean $=29.7$ years; $\mathrm{CI}_{95}=28.8$, $30.5)$ than $500 \mathrm{~T}$. gondii-negative primiparas $($ mean $=28.9$ years; $\left.\mathrm{CI}_{95}=28.5,29.2\right)$. This effect approached the level of significance $\left(\mathrm{F}=3.45, \mathrm{P}=0.064, \mu^{2}=0.006\right)$. The frequency of ART was $11.4 \%$ in the second data set; however, the influence of latent toxoplasmosis on the frequency of ART was not significant either in the all data set $(13.4 \%$ in T. gondii-positive mothers, $\mathrm{n}=201, v s 9.2 \%$ in $T$. gondiinegative mothers, $\mathrm{n}=815, \chi^{2}=1.01, \mathrm{P}=0.317, \mathrm{OR}=1.27$ ) or in primiparas $(15.9 \%$ in $T$. gondii-positive mothers, $\mathrm{n}=113$ vs $15.4 \%$, in $T$. gondii-negative mothers, $\mathrm{n}=500$; $\left.\chi^{2}=0.02, \mathrm{P}=0.888, \mathrm{OR}=1.04\right)$.

Differences in the time to conceive between T. gondiinegative and $T$. gondii-positive women were analysed for 317 respondents ( 75 were $T$. gondii-positive) who provided the information in the questionnaire study (study C). The Spearman correlation showed that $T$. gondii-positive women reported to take a longer time to conceive than T. gondii-negative women $(\mathrm{R}=0.14, \mathrm{P}=0.015$; Table 1$)$.

About 5000 subjects took part in the internet questionnaire study (study D). Among them, 524 women provided information about their T.gondii-infection status and rated their fertility problems on a 1-6 point scale (see Fig. 1). The association between toxoplasmosis and fertility problems was analysed with the partial Kendall's tau correlation test with maternal age as a confounding variable. The results showed that $154 T$. gondii-infected women reported more frequent or more serious fertility problems than 370 T. gondii-free women (partial Kendall's tau $=0.115$, $\mathrm{P}<0.0001)$.

\section{DISCUSSION}

Our results showed that pregnant women, especially primiparous, with latent toxoplasmosis were older than Toxoplasma gondii-negative pregnant women. Also, T. gondiipositive women reported to take a significantly longer time to conceive and to have more frequent or more serious fertility problems than $T$. gondii-free women. These results 


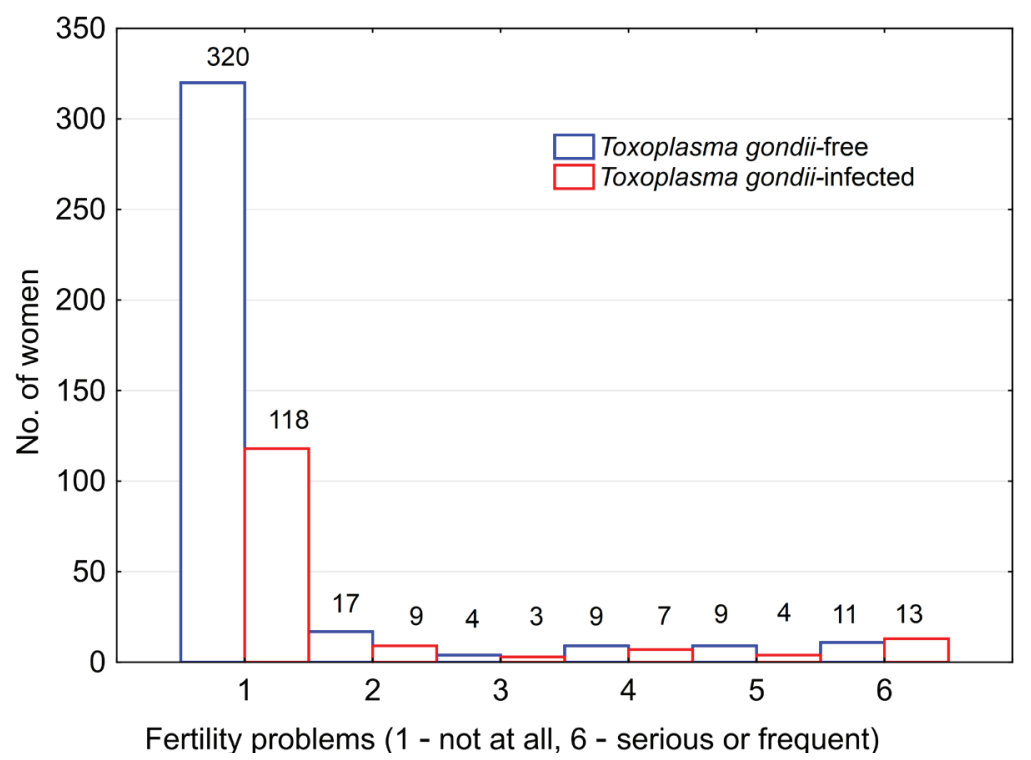

Fig. 1. Occurrence of fertility problems in women with and without latent toxoplasmosis (study D).

support the hypothesis that latent toxoplasmosis has some negative effects on the reproductive capacity of $T$. gondiiinfected women. Akarsu et al. (2011) have suggested that T. gondii-associated infertility mechanisms include development of endometritis and foetal rejection due to local release of $T$. gondii from cysts located in the endometrial tissue on stimulation during placenta formation, impaired folliculogenesis in the ovaries and uterine atrophy and reproductive failure due to hypothalamic dysfunction as a result of chronic toxoplasmosis. In mice, experimental data have shown acquired hypogonadotrophic hypogonadism secondary to hypothalamic dysfunction after chronic infection with T. gondii - Stahl et al. (1994), Antonios et al. (2000). However, the results of a study focused on T. gondii-seropositivity in fertile and infertile women have not indicated a relationship between toxoplasmosis and female infertility (Akarsu et al. 2011).

Toxoplasma gondii is a parasitic protozoan whose effects on human behavior (Flegr et al. 1996), personality (Lindová et al. 2006) and other phenotypic traits (Flegr 2013a) have been studied most thoroughly, often in the context of the manipulation theory. This theory suggests that many parasites change the phenotype of their host to increase their chances of transmission to a new host, for example by predation (Hutchison et al. 1980, Berdoy et al. 1995). The probability of congenital transmission in humans is very low, after tachyzoites of $T$. gondii transform to bradyzoites in tissue cysts. The intermediate host 'needs' to survive for as long as possible while $T$. gondii 'needs' the infected intermediate host to be killed and eaten by the definitive host (any feline species). It is possible that $T$. gondii manipulates the reproductive activity of an intermediate host (the vector) to increase its longevity and migratory activity at the expense of its reproduction rate. However, a more plausible explanation is that the observed association between toxoplasmosis and fertility problems is only a side-effect of the infection, e.g. the deteriorated health of chronically infected hosts.
Dvorakova-Hortova et al. (2014) have demonstrated a direct relation between infection with $T$. gondii and a decreased male reproductive fitness in mice. They have observed significant differences in the reproductive parameters obtained from a detailed testicular histometric analysis and in DNA methylation pattern of the testicular epigenome between $T$. gondii-infected and $T$. gondii-free mice. They have also observed a decreased level of luteinising hormone (LH) in urine of infected males. The urine LH level was studied to test the hypothesis that $T$. gondii might activate the hypothalamo-pituitary-adrenal (HPA) stress axis, thus modulating the hypothalamo-pituitary-gonadal (HPG) axis and consequently altering the release of gonadotropins (Dong et al. 2004, Terpsidis et al. 2009). Indirect indices of chronic stress in humans with latent toxoplasmosis have been reported by Lindová et al. (2010). These results, along with the evidence of negative effects of latent toxoplasmosis on health of mice (Flegr 2010) and humans (Flegr et al. 2014) are in agreement with our hypothesis that decreased fertility may be one of the side-effects of latent toxoplasmosis.

Latent toxoplasmosis leads to alterations in serum concentrations of testosterone both in men and mice (Flegr et al. 2008, Kaňková et al. 2011). Epidemiological and experimental evidence relates high concentrations of androgens to an increased risk of a reproductive failure (James 2015). It can be, therefore, speculated that toxoplasmosisassociated increases of testosterone could be the proximal cause of at least some maternal reproductive pathologies. It must be noted, however, that $T$. gondii-associated increase of testosterone was observed only in men and male rodents. The association between toxoplasmosis and fertility problems is thus rather a spurious one. Specifically, the women with higher level of steroid hormones could have both the fertility problems and also some immunity problems, which could result in an increased risk of protozoan infections. 
Studies performed in various countries have shown the existence of association between toxoplasmosis and mental health problems (Torrey et al. 2007, 2012, Holub at al. 2013, Flegr 2013b). We could speculate about the reasons for the correlation between the fertility and psychological problems of $T$. gondii-infected patients. It is possible that, at least in some subjects, the psychiatric disorders are direct consequences of the fertility problems. Many studies describe the increased prevalence of psychiatric disorders, e.g. depression, anxiety disorder (Volgsten et al. 2008) or psychosis, schizophrenia and other mental disorders in patients with fertility problems (undergoing in vitro fertilisation - IVF - treatment) (Baldur-Felskov et al. 2013). It is, however, also possible that the reported association between mental health and fertility problems is due to independent relations of mental health and fertility problems with $T$. gondii infection.

Our results showed that $T$. gondii-positive women used assisted reproductive technology to conceive significantly more often than $T$. gondii-negative women in the General University Hospital in Prague. However, this effect was not significant in women from outpatient clinics, Centre of Reproductive Medicine in Prague. This observation can be explained by the fact that women with medical problems and unsuccessful IVF trials are being referred to the public General Hospital from different Prague outpatient clinics.

Although both correlations, differences in the time to conceive between $T$. gondii-negative and T. gondii-positive women, and the association between toxoplasmosis and fertility problems are significant, the size effects are relatively low. This means that very little variability in the dataset can actually be predicted by $T$. gondii-status. La- tent toxoplasmosis therefore represents rather public health than individual subject-health problem.

An important limitation of studies $\mathrm{A}$ and $\mathrm{B}$ was the absence of information about the reasons for using ART in the available clinical records. Many women who used ART were in fact fully fertile and had partners with some fertility problems. In study D, women themselves reported their toxoplasmosis status. It is highly probable that some women provided incorrect information. Some of them, for example, may have tested negative several years ago but may have become infected in the meantime. It must be stressed out, however, that this kind of stochastic errors could increase the risk of false negative and not of false positive results of observational studies.

Our study provided several independent indices for the existence of an association between latent toxoplasmosis and fertility problems in women. The difficulties with conception represent a serious and growing public health problem in most of developed countries. Toxoplasmosis is the most widespread human parasitic infectious disease that affects about one third of the human population worldwide (Tenter et al. 2000). Currently, no method of treatment of latent toxoplasmosis is available. However, the prevalence of toxoplasmosis could be reduced by appropriate epidemiological approaches. Our results suggest that searching for anti- $T$. gondii vaccines for domestic cats as well as for a method of treatment of latent toxoplasmosis, is urgently needed.

Acknowledgements. The research was supported by research grants UNCE 204004 and PRVOUK 25 from the Charles University, Prague and RVO-VFN64165 from the Ministry of Health of the Czech Republic.

\section{REFERENCES}

Akarsu A.G., Elhan H.A., Akarsu C. 2011: Retrospective evaluation of Toxoplasma gondii seropositivity in fertile and infertile women. Mikrobiol. Bull. 45: 174-180.

Antonios S.N., Ismail H.I., Essa T. 2000: Hypothalamic origin of reproductive failure in chronic experimental toxoplasmosis. J. Egypt. Soc. Parasitol. 30: 593-599.

Baldur-Felskov B., KJaer S.K., Albieri V., Steding-Jessen M., Kuaer T., Johansen C., Dalton S.O., Jensen A. 2013: Psychiatric disorders in women with fertility problems: results from a large Danish register-based cohort study. Hum. Reprod. 28: 683-690

Berdoy M., Webster J.P., MacDonald D.W. 1995: Parasitealtered behaviour: is the effect of Toxoplasma gondii on Rattus norvegicus specific? Parasitology 111: 403-409.

Christiansen O.B., Pedersen B., Nielsen H.S., Andersen A.M.N. 2004: Impact of the sex of first child on the prognosis in secondary recurrent miscarriage. Hum. Reprod. 19: 2946-2951.

Dong Q., Salva A., Sottas C.M., Niu E., Holmes M., Hardy M.P. 2004: Rapid glucocorticoid mediation of suppressed testosterone biosynthesis in male mice subjected to immobilization stress. J. Androl. 25: 973-981.

Dvorakova-Hortova K., Sidlova A., Ded L., Hladovcova D., Vieweg M., Weidner W., Steger K., Stopka P., ParaDowsKa-Dogan A. 2014: Toxoplasma gondii decreases the reproductive fitness in mice. PLoS ONE 9: e96770.

FLEGR J. 2010: Influence of latent toxoplasmosis on the phenotype of intermediate hosts. Folia Parasitol. 57: 81-87.
FLEGR J. 2013a: Influence of latent Toxoplasma infection on the human personality, physiology, and morphology: pros and cons of the Toxoplasma-human model in studying the manipulation hypothesis. J. Exp. Biol. 216: 127-133.

FLEGR J. 2013b: How and why Toxoplasma makes us crazy. Trends Parasitol. 29: 156-163.

Flegr J., Hrdé Š., Kodym P. 2005: Influence of latent 'symptomatic' toxoplasmosis on body weight of pregnant women. Folia Parasitol. 52: 199-204.

Flegr J., Lindová J., Kodym P. 2008: Sex-dependent toxoplasmosis-associated differences in testosterone concentration in humans. Parasitology 135: 427-431.

Flegr J., Prandota J., SovičKová M., Israili Z.H. 2014: Toxoplasmosis - a global threat. Correlation between latent toxoplasmosis and specific disease burden - a WHO data-based study of 29 European and 59 non-European countries. PLoS ONE 9: e90203.

Flegr J., Zítková S., Kodym P., Frynta D. 1996: Induction of changes in human behaviour by the parasitic protozoan Toxoplasma gondii. Parasitology 133: 49-54.

Giorgino F.L., Mega M. 1981: Toxoplasmosis and habitual abortion. Our expedice. Clin. Exp. Obstet. Gynecol. 8: 132-134.

Holub D., Flegr J., Dragomirecká E., Rodriguez M., Preiss M., Novák M., Č́ermák J., Horáček J., Kodym P., Libiger J., Höschl C., Bankovská Motlová L. 2013: Differences in onset of disease and severity of psychopathology between toxoplasmosis-related and toxoplasmosis-unrelated schizophrenia. Acta Psychiat. Scand. 127: 227-238. 
Hostomská L., Jírovec O., HoráčKová M., Hrubcová M. 1957: Mongolismus und latente Toxoplasmosis der Mutter. Endokrinologie 34: 295-304.

Hutchison W.M., Bradley M., Cheyne V.M., Welh B.W.P., HaY J. 1980: Behavioural abnormalities in Toxoplasma-infected mice. Ann. Trop. Med. Parasitol. 74: 337-345.

JAMES W.H. 2015: High levels of maternal adrenal androgens are a major cause of spontaneous abortion and other forms of reproductive sub-optimality. J. Theor. Biol. 364: 316-320.

KAŇKOVÁ Š., FLEGR J. 2007: Longer pregnancy and slower fetal development in women with latent 'asymptomatic' toxoplasmosis. BMC Infect. Dis. 7: 114.

KaňkovÁ Š., Kodym P., Flegr J. 2011: Direct evidence of Toxoplasma-induced changes in serum testosterone in mice. Exp. Parasitol. 128: 181-183.

Kaňková Š., Kodym P., Frynta D., Vavřinová R., Kuběna A., FLEGR J. 2007b: Influence of latent toxoplasmosis on the secondary sex ratio in mice. Parasitology 134: 1709-1717.

Kaňková Š., Procházková L., Flegr J., Calda P., Springer D., Potluková E. 2014: Effects of latent toxoplasmosis on autoimmune thyroid diseases in pregnancy. PLoS ONE 9: e110878.

KaňKová Š., Šulc J., Nouzová K., Fajfrlík K., Frynta D., FLEGR J. 2007a: Women infected with parasite Toxoplasma have more sons. Naturwissenschaften 94: 122-127.

Kellokumpu-Lehtinen P., Pelliniemi L.J. 1984: Sex ratio of human conceptuses. Obst. Gynecol. 64: 220-222.

Kimball A.C., Kean B.H., Fuchs F. 1971: The role of toxoplasmosis in abortion. Am. J. Obsest. Gynecol. 111: 219-226.

Kodym P., Machala L., Roháčová H., Širocká B., Malý M. 2007: Evaluation of a commercial IgE ELISA in comparison with IgA and IgM ELISAs, IgG avidity assay and complement fixation for the diagnosis of acute toxoplasmosis. Clin. Microbiol. Infec. 13: 40-47.

Lindová J., KubĚna A.A., Šturcová H., Křivohlavá R., Novotná M., Rubešová A., Havlíček J., Kodym P., Flegr J. 2010: Pattern of money allocation in experimental games supports the stress hypothesis of gender differences in Toxoplasma gondii-induced behavioural changes. Folia Parasitol. 57: 136142.
Lindová J., Novotná M., Havlíček J., Jozífková E., SKallová A., Kolbeková P., Hodný Z., Kodym P., Flegr J. 2006: Gender differences in behavioural changes induced by latent toxoplasmosis. Int. J. Parasitol. 36: 1485-1492.

Pavlinová J., Kinčeková J., Ostró A., Saksun L., Vasilková Z., KöNIGOVÁ A. 2011: Parasitic infections and pregnancy complications. Helminthologia 48: 8-12.

Quablan H.S., Jumaian N., Abu-Salem A., Hamadelil F.Y., Mashagbeh M., Abdel-Ghani F. 2002: Toxoplasmosis and habitual abortion. J. Obstet. Gynaecol. 22: 296-298.

Salman Y.J. 2014: Correlation between Toxoplasma gondii and Anti-Mullerian hormone levels in sera of women in Kirkuk City using ELISA method. Int. J. Curr. Microbiol. Appl. Sci. 3: 85-92.

Siegel S., Castellan N.J. 1988: Nonparametric Statistics for the Behavioral Sciences. Second Edition, McGraw-Hill, New York, $399 \mathrm{pp}$.

Stahl W., Kaneda Y., Noguchi T. 1994: Reproductive failure in mice chronically infected with Toxoplasma gondii. Parasitol. Res. 80: 22-28.

Tenter A.M., Heckeroth A.R., Weiss L.M. 2000: Toxoplasma gondii: from animals to humans. Int. J. Parasitol. 30: 1217-1258.

Terpsidis K.I., Papazahariadou M.G., Taitzoglou I., PaPaioannou N.G., Georgiadis M.P., Theodoridis IT. 2009: Toxoplasma gondii: reproductive parameters in experimentally infected male rats. Exp. Parasitol.121: 238-241.

Torrey E.F., Bartko J.J., Lun Z.R., Yolken R.H. 2007: Antibodies to Toxoplasma gondii in patiens with schizophrenia: a meta-analysis. Schizophr. Bull. 33: 729-736.

TorRey E.F., Bartko J.J., Yolken R.H. 2012: Toxoplasma gondii and other risk factors for schizophrenia: an update. Schizophr. Bull. 38: 642-647.

Volgsten H., Svanberg A.S., Ekselius L., Lundkvist Ö., PoROMAAL I.S. 2008: Prevalence of psychiatric disorders in infertile women and men undergoing in vitro fertilization treatment. Hum. Reprod. 23: 2056-2063.

Wolf A., Cowen D., Paige B.H. 1939: Toxoplasmic encephalomyelititis. III. A new case of granulomatoces encephalomyelititis due to a protozoon. Am. J. Pathol. 15: 657.

Cite this article as: Kaňková Š., Flegr J., Calda P. 2015: The influence of latent toxoplasmosis on women's reproductive function: four cross-sectional studies. Folia Parasitol. 62: 041. 